\title{
On Ancient Chinese Translation
}

\author{
Siyu Zou \\ Nanchang Institute of Technology
}

\begin{abstract}
Keywords: Ancient prose; Ancient prose features; Text; English translation
\end{abstract}
\begin{abstract}
With the accelerated process of economic globalization, people's desire to know foreign culture is also growing. As one of cross-cultural communication bridges, translation draws more and more attention of people. Translation is not only to carry out the conversion of language symbols, but also to convert the culture in a language into another culture form. Translation has become a communication activity crossing language, culture and society. Ancient Chinese language is like fog with a ray of light guiding readers. During the walk, readers may see different scenery. In the process, the author may gently sigh tell in readers' ears. Some have no word forms but word conception; some have word forms but no word meanings. How to dispel the clouds and see the sun in the process of translation, and give readers are not familiar with Chinese culture the closest and the original language. This paper will analyzes the characteristics of the translation process of Chinese ancient books, including the popularization of understanding and expression, the conversion of formation and parataxis, and the translation of idioms.
\end{abstract}

\section{Introduction}

The Chinese nation has a very splendid culture, leaving the colorful voluminous ancient books, such as poetry, prose, fiction and drama. With the increasing exchanges of Eastern and Western culture, the ancient Chinese prose are also more and more translated into English and spread in the Western world where both the thinking and language are very different from the Eastern world. An important prerequisite for the translation of classical Chinese into English is translators' understanding of Chinese classical works. Translators' grasp to the original meaning may decide the translation of texts. In ancient classical Chinese, sublime words always have deep meaning, which requires translators use their own aesthetic consciousness and cultural awareness to understand and interpret. If translators have no correct understanding, it is impossible to have correct translation. Classical Chinese is the treasure of China, so it is necessary to have good translation principles. In this paper, the significance and the present situation of the translation of ancient Chinese are discussed, and then the characteristics of ancient books are explained. Finally, some examples are given in ancient text translation. From above, we can understand the translation principles which will be good reference for translators. This paper tries to discuss some basic rules in English translation according to the stylistic features of Chinese classical literature and asks advice for readers.

\section{The Significance and Present Situation of the English Translation of Ancient Books}

The Chinese civilization is long and glorious. Sages left numerous eternal beauty and wisdom and the immortal works which are worth reading again and again. When talking about Chinese culture, Analects of the Confucius must be mentioned. His mind is like the fertile soil for root germination of Chinese culture and he has influence both at home and abroad both in history and at present as one of the bright pearl in Chinese cultural heritage.

English is widely used in the world. Classical translation, which is to convert Chinese books into English, naturally constitutes the important aspects of cultural communication. To read these classical books in another language may bring readers another flavor, maybe other inspiration. Classical translation has great practical significance for developing national culture, integrating Eastern and Western culture and maintaining the inherent in the Chinese cultural identity,

The present situation of English translation of classical ancient books is that the translation is difficult, and that of many other ancient works, such as Analects of Confucius, the translation is 
more difficult. English and Chinese are two quite different language independent systems. When the same content is expressed in English and Chinese respectively, the sequence of the component information will inevitably vary and may sometimes the different is even very large. The present situation and problems of the English translation of Chinese classics is due to language barriers and other reasons. Chinese culture is still not fully understand in the world. Guan Hanqing has more works than Shakespeare does, but the world knows Shakespeare better than Guan, which illustrates that the cultural dissemination in China is weak and is not conducive to the cultural exchanges between China and foreign countries.

\section{Characteristics of Chinese Ancient Books}

With the integration time and culture, there are a lot of differences between ancient and modern Chinese. Firstly, Chinese ancient prose has no punctuation, that is to say the logo of projection quotes does not exist. Translators have to add by their own in the process of translation. The different word segment in the wording of the sentence may be different in translation. But the treasure of Chinese shall be seen by the world. Some things are easier to translate, and even can be summed up in some basic framework to save time and energy. But there is no limit to the improvement of some things. The indifferent landscape gardening of the philosophy in Chinese culture is difficult to be fully understood or appreciated in western context.

In Chinese ancient book, a lot of China reasoning and narrative process are completed in the form of dialogues. In many narrative genre, the authors themselves rarely express their thoughts directly, and often use other people's statements, or other people's reactions. For example, the evaluation of Zhou Chu and the dialogues in Strategies of the Warring States, are sued to show the counsel and the words of the lobbyists. In the lyrics and other major literary works, a "cipai" (names of the tunes to which a poem is composed or a song is often used to show the development track of the story and to complete the characters portray, such as Palace of Eternal Life. In Confucian classics Analects, there are many reasoning and evaluation, which are all shown through the dialogue between the characters. In this colorful literature atmosphere, if translators blindly translate the Chinese character "Yue"into "say", it will be eventually not very appropriate. The following are some analysis for specific problems.

\section{English Translation of Classical Chinese}

In the translation of classical Chinese, the above stylistic characteristics of the classical Chinese need to be emphasized, we also need to combine the characteristics of English, preserve the original meaning and style at the same time, take care of the reading habits of target language readers and try to make a balance between the two languages. Based on this, the translation of classical Chinese can be performed in the following several aspects:

1. Tell the meaning of a word, pay attention to words

Because in classical Chinese literature, there are many monosyllabic words and there are many words that have more than one use. So in translation, the appropriate words shall be chosen according to the context and the meaning, for example:

(1) La yue,Chen yu zhi nu yin,hai zhi xia cheng fu.(History Records: Chen Family)

In the twelfth month, Chen She went to Ruyin.

"Zhi" means "Wang", so it is translated into "went to".

(2) Min ri, xu gong lai, shu shi zhi, zi yi wei bu ru。(Strategies of the Warring States: Self-knowledge Topics Thorough Understanding of Their Own)

The next day Lord Xu himself called and Zou Ji, examing him carefully, suspected that he was outmatched.

Here, "zhi" refers to a person, so it is translated into "him".

(3) Mu qin er si zhi。(the same as above)

In bed that night he thought the matter over.

Here, "Zhi" refers to matter. 
In the three cases, “之” has three different meanings although it is in the same form. So, it should be selected the English words based on its practical significance.

2. Identify the meaning and choose the part of speech.

There are many words that have more than one use in ancient Chinese. In English translation, corresponding conversion of part of speech shall be done according to the practical needs.

(1) Chu zuo yin bo zhe, xiang yu ji fu ye, su shan liu hou zhang liang。(History Records: A Dinner at Hongmen)

"Shan" is an adjective, in this example, it is used as verb. Attention shall be paid in translation. The sentence shall be translated into "Be a friend of".

Xiang Yu's uncle, Xiang Bo , the left minister of Chu, was a friend of Zhang Liang.

(2) Dang ci shi, zhu jun xian ku qin li zhe,jie xing qi chang li sha zhi yi ying chen she。

"Ku" is an adjective or noun, in this example, it is used as verb. "Xing" is a noun, in this example, it is used as verb. Attention shall be paid in translation.

Thereupon all in the provinces and countries who hated the rule of Qin rose against their governors and magistrates and killed them in order to follow Chen She's lead.

3. Omitting Translation

In ancient Chinese, some structure or tone of the auxiliary table shall be omitted when translated into English, because English sentence features imply the framework; or because English sentences are unfavorable expression the tone.

(1)Chen sheng zhe, yang cheng ren ye, zi she。(History Records: Chen Family)

Chen Sheng, whose formal name was She was a native of Yongchen.

(2)Wei zhi ming nian you zai he chu, qi ju zhu lou zhi yi xiu hu? (The Bamboo Pavilion in Huanggang by Wang Yu)

I have no idea where I shall be next year, why ,then should I worry because this Bamboo Pavilion may not last?

(3)Qian ren zhi shu bei yi。 (Notes on the Yueyang Tower by Fan Zhongyan)

All this has been described in full by writers of earlier ages

4. Adding Translation

Subject or implicit association words shall be paid attention to be added in translation from classical Chinese text into English.

(1)You za zhi lan gui zhu mu yu ting, jiu shi lan dun,yi sui zeng sheng。(Nape by Gui Youguang)

I also planted orchids, cassias and bamboos, showing off the old balustrades to better advantage.

In this case, the subject is missing and shall be added in English translation.

(2)Sheng ren wu chang shi, Kongzi shi tan zi 、 chang hong、 shi nang、 lao dan。 (Han Yu: Of Teachers)

A sage has more than one teacher, thus Confuius learned from Tan Zi, ChangHong, Shi Xiang and Lao Dan.

In this translation, the hidden associated words "thus" and "and" shall be added.

There are many other stylistic characteristics of ancient Chinese prose, this paper just tries to analyze the prominent ones, especially the ones relating to English language with the hope of being the reference for the future translation.

\section{Conclusion}

There is a statement "gu tian xia zhi dong zhe cun hu ci" which means to describe the changes of the world through the language in The Book of Changes. As the symbol of thoughts, it is no doubt that language contains tremendous strength. In the process of translation, how to transfer the power completely a major problem faced by English learners. Especially in the translation of ancient Chinese. Since the ancient Chinese works are of old age, many concrete has no textual criticism, which is indeed a big challenge for the scholars who are learning English. What's more, in ancient books, both astronomy, geography, literature, history and philosophy are often involved, which has 
high requirements for translators. But in translation, there is no disciplinary boundaries, which is the so-called "bypass without delay, but not daily".

\section{References}

[1] Ming-Dong L I, University J. The Influence of Chinese Parataxis on the Translation Strategy of Ancient Chinese Poetry into English [J]. Journal of Chongqing University of Technology, 2013.

[2] Wang X. Five Sets of Categories, Four Sets of Rules and Structure of Ancient Chinese Translation Studies [J]. Journal of Anhui University, 2016.

[3] Wang S, Xiong D L, Wang X X. The Design and Implementation of Ancient Chinese Translation System [J]. Computer Knowledge \& Technology, 2009, 1:194-198.

[4] Gao L T. Ancient Indian Marriage Customs in Buddhist Precepts of Chinese Translation [J]. Journal of Lvliang University, 2012.

[5] Tong L. A research on the Chinese translation of ancient Indian Serpent demons NÄ• ga[J]. Journal of Sino-Western Communications, 2010.

[6] Ding Y, Li R, Li W. Ancient Chinese musical score translation via instance-based learning[C]// International Conference on Audio, Language and Image Processing. 2012:1035-1040.

[7] Suqing H U. Ancient Chinese as "a Process Language": Roger Ames' View of Chinese and Translation Practice [J]. Journal of Central South University, 2013.

[8] $\mathrm{Pu} \mathrm{H}$. The Influence of Chinese Translation of Buddhist Scriptures and Its Philosophy on Ancient Chinese Poetics [J]. Literature \& Art Studies, 2005.

[9] Cao X Y. Early Chinese Translation of Buddhist Sutra and the Lexical Etymologies of Middle Ancient Chinese Language Words and Expressions [J]. Journal of Hefei Normal University, 2010.

[10]Qui G, Kashima J. Translation from Chinese: Ancient Chinese Weights and Measures [J]. Bulletin of the Society of Historical Metrology, Japan, 2000, 22:77-96.

[11]John M. I Ching: The Essential Translation of the Ancient Chinese Oracle and Book of Wisdom $[\mathrm{J}]$.

[12]Huang Z L. On Transforming Mechanism of Chinese Ancient Books and Records' Translation [J]. Yuejiang Academic Journal, 2012. 